\title{
Article \\ Numerical Study of the Improvement in Stability and Performance by Use of a Partial Vaned Diffuser for a Centrifugal Compressor Stage
}

\author{
Shuai $\mathrm{Li}^{1}{ }^{1}$, Yan Liu ${ }^{1, *}$, Hongkun $\mathrm{Li}^{2}$ and Mohammad Omidi ${ }^{1}$ \\ 1 School of Energy and Power Engineering, Dalian University of Technology, Dalian 116024, China; \\ lishuai17@mail.dlut.edu.cn (S.L.); Omidi@dlut.edu.cn (M.O.) \\ 2 School of Mechanical Engineering, Dalian University of Technology, Dalian 116024, China; lihk@dlut.edu.cn \\ * Correspondence: yanliu@dlut.edu.cn
}

Citation: Li, S.; Liu, Y.; Li, H.; Omidi,

M. Numerical Study of the

Improvement in Stability and

Performance by Use of a Partial

Vaned Diffuser for a Centrifugal

Compressor Stage. Appl. Sci. 2021, 11,

6980. https://doi.org/10.3390/

app11156980

Academic Editor: José A.F.O. Correia

Received: 1 July 2021

Accepted: 26 July 2021

Published: 29 July 2021

Publisher's Note: MDPI stays neutral with regard to jurisdictional claims in published maps and institutional affiliations.

Copyright: (c) 2021 by the authors. Licensee MDPI, Basel, Switzerland. This article is an open access article distributed under the terms and conditions of the Creative Commons Attribution (CC BY) license (https:/ / creativecommons.org/licenses/by/ $4.0 /)$

\begin{abstract}
The influence of different diffuser configurations on the flow stability and aerodynamic performance of a centrifugal compressor stage with a mass flow coefficient of 0.196 is numerically investigated. Research results show that the performance of a traditional full-height vaned diffuser (TVD) deteriorates rapidly, and a shroud-side partial vaned diffuser (SVD) displays better adaptability in off-design conditions. SVD can suppress the development of vortices generating at the diffuser leading-edge. Therefore, it can reduce the flow loss inside the stage and improve the flow stability of the stage at low mass flow rates. The unsteady analysis for TVD and SVD shows that the stall cell propagates at about $35.7 \%$ of impeller rotational speed in the semi-vaneless space and diffuser passages. Furthermore, the internal flow in TVD and SVD is studied by employing the proper orthogonal decomposition (POD) and dynamic mode decomposition (DMD) methods. The flow loss and instability mechanism in the stage are consequently revealed more comprehensively.
\end{abstract}

Keywords: partial vaned diffuser; flow stability; compressor stage; unsteady analysis; proper orthogonal decomposition

\section{Introduction}

With the large-scale development of process equipment in the petrochemical and coal chemical industries, the demand for centrifugal compressors for large-scale plants is also increasing. It is vital to the manufacturing cost, energy consumption, and compact structure to reduce the size of centrifugal compressors stages. At present, increasing the flow capacity of a single stage of a centrifugal compressor (i.e., increasing the design flow coefficient) is a very effective method. However, problems such as low efficiency and narrow operating ranges can arise. These problems are closely related to sizeable impeller-exit width and the unstable flow inside the centrifugal compressor with a large mass flow coefficient, which restricts broader applications of the large mass flow coefficient compressor.

To improve the aerodynamic performance of a compressor stage, a good diffuser becomes essential in homogenizing the flow field and efficiently recovering the kinetic energy of fluid from the impeller exit. A vaneless diffuser (VD) is widely used in centrifugal compressors for its low costs and wide operating ranges. However, a large radius ratio will cause severe flow loss. A traditional full-height vaned diffuser (TVD) can provide better aerodynamic performance than a VD near the design point. However, if the operation deviates from the design point, the throat limits the operating range of the compressor [1]. To solve this problem, a partial vaned diffuser can be used. The advantage of the partial vaned diffuser is the integration of VD and TVD. The performance of the diffuser is related mainly to the flow condition at the impeller exit, and the performance of the impeller is also affected by the diffuser [2], especially the complex impeller exit flow of a large mass flow coefficient compressor. To understand the matching of diffuser and impeller, it is necessary to study different types of diffusers. 
Issac et al. [3] changed the blade height of partial vaned diffusers and compared the aerodynamic performance. Research shows that there is an optimum vane height for each partial vaned diffuser. The application of a partial vaned diffuser can effectively suppress the corner separation flow, as demonstrated by Yoshinaga et al. [4]. Based on experimental and simulation results, Zhang et al. [5] and Zhao et al. [6] pointed out that centrifugal compressors have higher stage performance when assembled with shroud-side partial vanes. In contrast, Liu et al. [7] found that a hub-side vaned diffuser has higher pressure recovery and efficiency than a vaneless diffuser and has a wider operating range compared with a traditional vaned diffuser. Sitaram et al. [8] showed that a hub-side vaned diffuser has a higher energy coefficient and a wider operating range under a certain vane height. The experimental and simulation investigations by Ohta et al. [9] indicated that the tapered vaned diffuser could reduce noise and enlarge the stable operating ranges by vortex control. Although numerous studies show that a partial vaned diffuser can efficiently improve the internal flow of the compressor, the conclusions about the arrangement of partial vanes as well as the details of the internal flow of the diffuser are somewhat contradictory. Moreover, the loss mechanism and dynamic characteristics of flow instability need further investigation. Therefore, this paper attempts to provide a comprehensive representation of the flow pattern of a centrifugal compressor assembled with different types of vaned diffusers to explore various flow losses and mechanisms of stability improvement.

Firstly, detailed configurations of the diffuser and the numerical method are presented, and experimental data authenticate the accuracy of the numerical method. Then, according to unsteady time-averaged results, the performance and internal flow of different diffusers are investigated. Moreover, unsteady numerical simulations for TVD and SVD under low mass flow rate are carried out. The evolution process of the main vortex structures of the leading edge of diffuser vanes and the propagation law of the stall cell are described in detail. Finally, the large-scale flow structure in the diffuser passage for TVD and SVD is analyzed utilizing the proper orthogonal decomposition (POD) and dynamic mode decomposition (DMD) methods.

\section{The Centrifugal Compressor Stage Investigated}

The centrifugal compressor stage selected for the current study represents the existing industrial centrifugal compressor, which consists of a backswept shrouded impeller, a diffuser, and a return channel. The design mass flow coefficient $(\varphi)$ of the compressor stage studied is 0.196 , and the design machine Mach number $M_{\mathrm{u} 2}$ is 0.9 corresponding to the rotational speed of $16,382 \mathrm{rpm}$. Due to the power limitation of the test rig used, the maximum machine Mach number $M_{\mathrm{u} 2}$ of 0.8 corresponding to the rotational speed of $14,562 \mathrm{rpm}$ (about $88.9 \%$ design rotation speed) was reached. To compare with the experimental data, numerical simulations in this paper applied the same operating conditions as the experiment. The impeller includes 17 blades. The diameter of the impeller exit is $360 \mathrm{~mm}$. The return channel consists of 18 blades [6]. The primary geometric parameters of the centrifugal compressor stage are shown in Table 1. Four types of diffusers were investigated. Three of them were vaned diffusers, and each included 22 vanes. The fourth was a vaneless diffuser. The diffusers had an identical meridional profile.

Table 1. Primary geometric parameters of the centrifugal compressor stage.

\begin{tabular}{ccc}
\hline Parameters & & Value \\
\hline Impeller exit diameter & $D_{2}$ & $360 \mathrm{~mm}$ \\
Diffuser entrance diameter & $D_{3}$ & $390 \mathrm{~mm}$ \\
Diffuser exit diameter & $D_{4}$ & $465 \mathrm{~mm}$ \\
Impeller exit width & $b_{2}$ & $17 \mathrm{~mm}$ \\
Number of impellers & $Z_{\text {imp }}$ & 17 \\
Number of diffuser vanes & $Z_{\text {dif }}$ & 22 \\
Number of return channels & $Z_{\mathrm{r}}$ & 18 \\
Rotational speed & $\omega$ & $14,562 \mathrm{rpm}$ \\
\hline
\end{tabular}


Three different vaned diffusers were selected for this study: a traditional full-height vaned diffuser (TVD), a shroud-side partial vaned diffuser (SVD), and a hub-side partial vaned diffuser (HVD). The vane height of SVD/HVD is about $21.4 \%$ of the TVD vane height. Our team carried out a numerical design and investigation for the vane height of a partial vaned diffuser [6]. The geometries of different types of diffusers are presented in Figure 1.

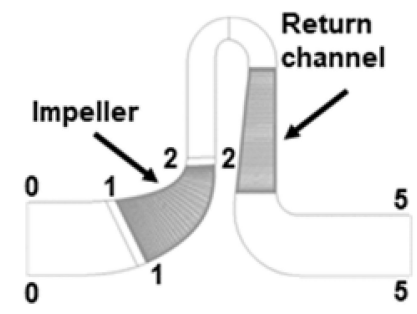

(a)

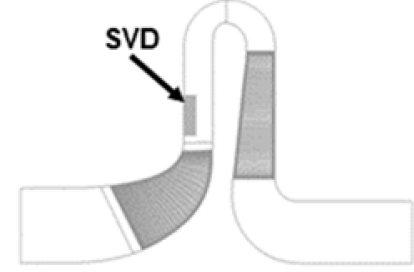

(c)

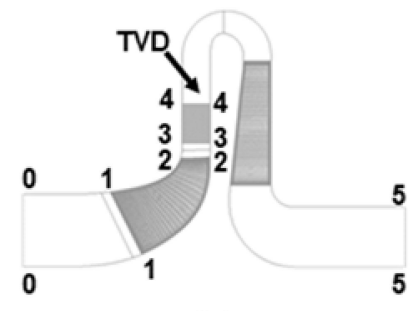

(b)

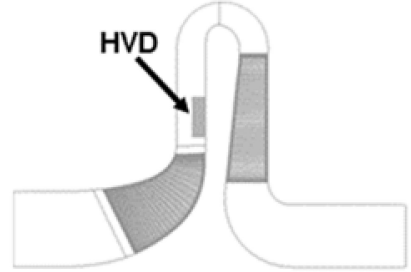

(d)

Figure 1. Meridional schematic of diffuser: (a) VD; (b) FVD; (c) SVD; (d) HVD.

\section{Methodology}

\subsection{Computational Modeling and Grid}

Figure 2 displays the three-dimensional numerical model (SVD). Considering the accuracy and cost of numerical simulations, this study adopts the same method as $[10,11]$, namely the multipassage calculation model, which includes three impeller passages, three diffuser passages, and three return channel passages for VD case and three impeller passages, four diffuser passages, and three return channel passages for TVD, SVD, and HVD cases, as shown in Figure 2a.

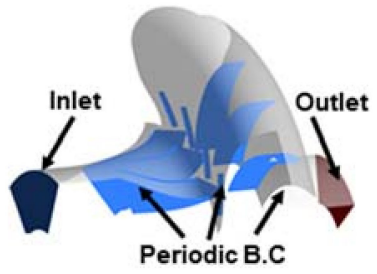

(a)

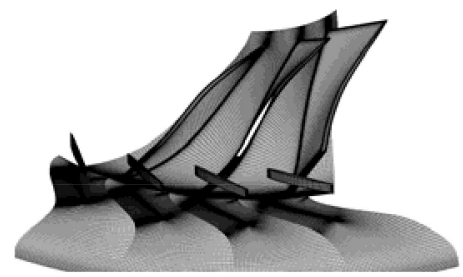

(b)

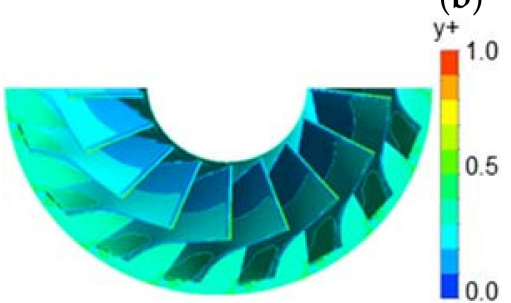

(c)

Figure 2. Computational domain and grid: (a) multi-passage model; (b) grid; (c) y plus.

All computational domains employ multiblock structured hexahedral meshes generated by the commercial code NUMECA/Autogrid. Figure $2 b$ shows the computational grid for the impeller and diffuser passages. The grids near the end walls are refined to capture more flow details, which is crucial for compressible flows [11]. Therefore, high 
precision and meticulous care are exerted by controlling grid points close to the end walls to ensure sufficient resolution for compressible flow calculation. The first cell height is set to $10^{-6} \mathrm{~m}$ corresponding to the dimensionless wall distance $\mathrm{y}+$ below unity along all end walls (design point flow rate and low flow rate conditions, Figure 2c). The impeller passage grid contains 77, 165, and 82 points in the spanwise direction, streamwise direction, and pitchwise direction, respectively. The vaned diffuser passage grid contains 73, 169, and 141 points in the spanwise direction, streamwise direction, and pitchwise direction, respectively [12]. The maximum mesh skewness is about 0.63 , which meets the grid accuracy to resolve the secondary flow. Considering the accuracy of computation results, a grid independence study was conducted using four different grids, fine, medium 1, medium 2 , and coarse. The total pressure ratio $(\varepsilon)$ for the impeller and the static pressure recovery coefficient $\left(C_{\mathrm{p}}\right)$ for the vaned diffuser are taken as parameters between different grids. The difference between the medium 2 grid and the fine grid was less than $0.16 \%$ for the impeller and diffuser, as shown in Table 2. A similar analysis was performed for the VD, TVD, and HVD. Therefore, the medium 2 grids were selected for the simulations. In the computational model, the inlet extended domain, impeller domain, and return channel domain have 206,625, 1,367,430, and 2,585,178 nodes, respectively. VD, TVD, SVD, and HVD have 276,507, 3,193,312, 3,244,068, and 3,244,068 nodes, respectively.

Table 2. Research on grid independence for impeller and SVD domain.

\begin{tabular}{cccc}
\hline Component & & $\boldsymbol{\varepsilon}$ & Difference \\
\hline \multirow{2}{*}{ Impeller } & Coarse & 1.3707 & $1.182 \%$ \\
& Medium 1 & 1.3796 & $0.5407 \%$ \\
& Medium 2 & 1.3853 & $0.1298 \%$ \\
& Fine & 1.3871 & - \\
\hline \multirow{2}{*}{ SVD } & & $\boldsymbol{C}_{\mathrm{p}}$ & Difference \\
& Coarse & 0.3782 & $1.843 \%$ \\
& Medium 1 & 0.3831 & $0.571 \%$ \\
& Medium 2 & 0.3847 & $0.1557 \%$ \\
\end{tabular}

\subsection{Numerical Scheme}

ANSYS CFX 18.2 was used to calculate the internal flow in the centrifugal compressor stage. The 3D (U)RANS equations and the energy equation for ideal gas are solved by a coupled implicit pressure-based solution technique. Advection and diffusion fluxes in the governing equations are discretized utilizing a "High Resolution" advection scheme, which is a second-order scheme and bounded [6]. All walls of the centrifugal compressor stage are assumed to be adiabatic, smooth, and no-slip. The working medium is an ideal gas. Considering the consistency with the experimental measurement, total temperature $(293 \mathrm{~K})$ and total pressure $(98,000 \mathrm{~Pa})$ are set at the stage entrance. The boundary condition of stage exit is the mass flow rate. The shear stress transport (SST) turbulence model is chosen to close governing equations.

For the unsteady simulations, a transient rotor-stator interface is used for the inlet duct-impeller and impeller-diffuser interface. The transient scheme is employed in the second-order backward Euler scheme. The unsteady simulation can approximately simulate the real internal flow of the computational model.

Time-dependent behavior for unsteady simulations is characterized through time step $(\Delta t)$ and time duration. The time step is related to the angular displacement (rotational speed) of the impeller [13]. To ensure the accuracy of transient simulations, the influence of time step on transient simulations is investigated. Figure 3 displays the time evolution of the static pressure coefficient for one designated point in SVD with three transient time steps. It is observed that the differences in the results are insignificant as time steps are increased. Considering the solution accuracy and simulation time, $\Delta t=1.15413 \times 10^{-5} \mathrm{~s}$ is chosen, which corresponds to a rotation of 1 degree of the impeller. In each transient simulation, the 
internal flow in the compressor reaches periodic fluctuations and then calculates several revolutions to obtain a more accurate internal flow field. A high-performance parallel workstation was used for numerical simulations, and the transient simulation took about 15,000 CPU hours.

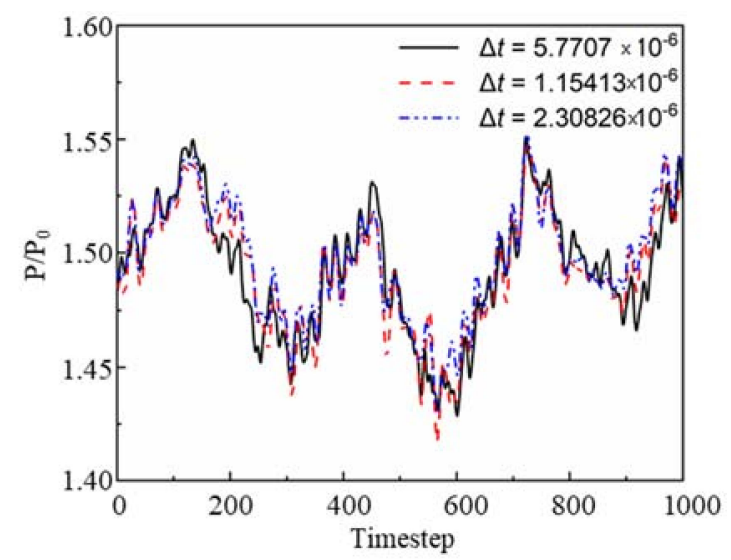

Figure 3. Time evolution of pressure coefficient with three different time steps for SVD configuration.

\subsection{Modal Decomposition Methods}

To further study the unsteady events in the centrifugal compressor stage, two modal decomposition methods (POD and DMD) were applied to extract the large-scale flow structures from the massive computation data. The POD method was first proposed by Lumley [14] to distinguish the large-scale flow structures in turbulent flows. The principal objective of the POD method is to approximately describe the high-dimensional data with low-dimensional data and then achieve dimensionality reduction. With the improvement of the POD algorithm, the snapshot-POD method [15] is applied, which greatly reduces the computational effort of the POD method. Moreover, algorithm procedures can be found in the works of Sirovich et al. [15,16], Kim et al. [17], and Shi et al. [18]. The POD method cannot identify the flow phenomenon at only one specific frequency. The DMD method can identify the flow at a single frequency, which complements the analysis of the POD method. The algorithm procedures of the DMD method can be found in the work of Schmid [19]. Therefore, the snapshot-POD method and DMD method were employed to estimate the unsteady flow field inside the centrifugal compressor.

\section{Results and Discussion}

This section is organized into the following parts: the first one presents the timeaveraged unsteady simulation results, including numerical method validation, aerodynamic performance, and internal flow of different diffusers. Then, the unsteady simulation results of TVD and SVD at the stall point are presented to describe the internal flow and transient instability evolution processes of the vaned diffuser. Finally, results of the analysis of the large-scale flow structure in TVD and SVD by the POD and DMD methods are presented.

\subsection{Time-Averaged Unsteady Simulation Results \\ 4.1.1. Numerical Method Validation}

The performance of SVD was measured in the centrifugal compressor test facility of the Shenyang Blower Works Group Corporation. The structure of the test rig is shown in Figure 4. More details about the measuring instruments, data acquisition, and experimental accuracy apparatus are given in [20]. 


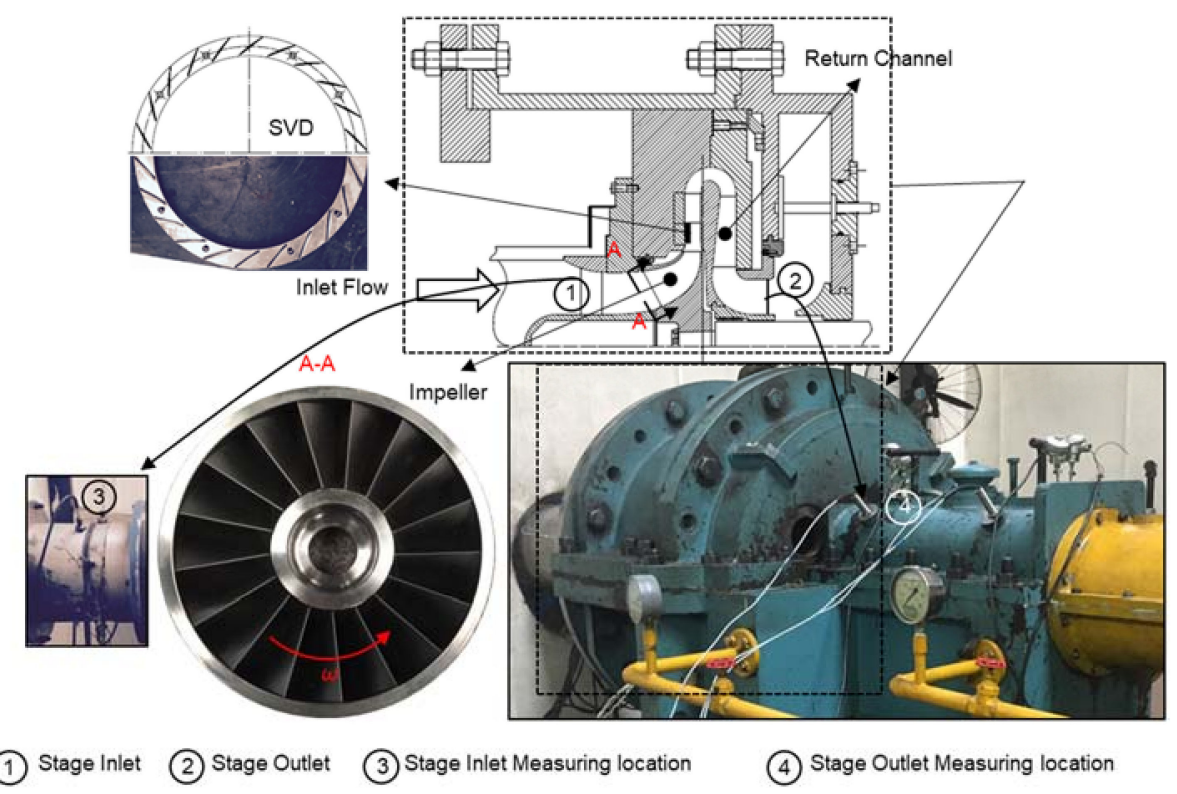

Figure 4. Schematic sketch of the test rig.

To verify the current numerical methods, time-averaged unsteady simulation results are compared with the experimental measurement data. Figure 5 compares the predicted results with the measured performance results for SVD. The abscissa is the flow coefficient normalized by the design mass flow rate, while the ordinate is the total pressure ratio $\varepsilon$ and total-total polytropic efficiency $\eta_{\text {pol }}$. Parameter $\varepsilon_{05}$ is the ratio of the total pressure mass-averaged on the cross-section at the compressor stage exit to that at the stage entrance; i.e., $\varepsilon_{05}=P_{\mathrm{t} 5} / P_{\mathrm{t} 0}$. The $\eta_{\mathrm{pol}}$ is given in Equation (1):

$$
\eta \text { pol }=\frac{\kappa-1}{\kappa} \frac{\ln \frac{P_{\mathrm{t} 5}}{P_{\mathrm{t} 0}}}{\ln \frac{T_{\mathrm{t} 5}}{T_{\mathrm{t} 0}}}
$$

where $\kappa$ is the isentropic exponent, which is equal to 1.4 , and $T_{\mathrm{t} 0}$ and $T_{\mathrm{t} 5}$ are the total temperatures mass-averaged on the cross-sections at the compressor stage inlet and outlet, respectively.

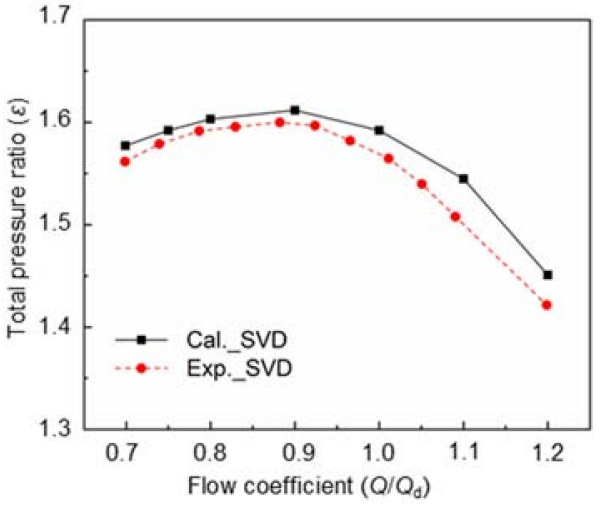

(a)

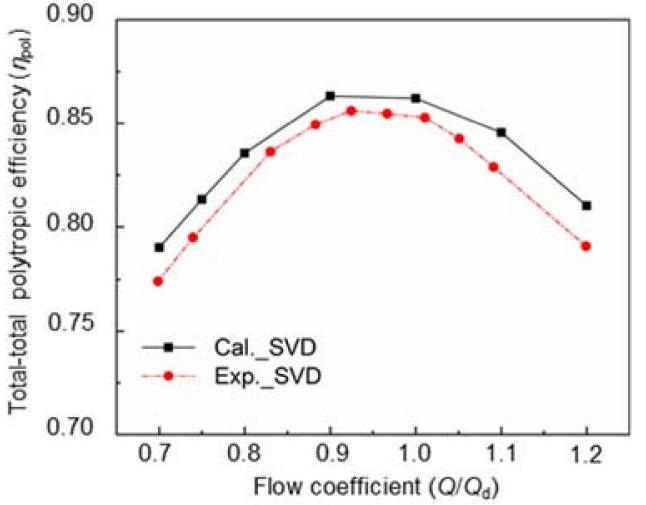

(b)

Figure 5. Comparison of CFD-predicted results with experimental data for the stage with SVD: (a) total pressure ratio; (b) total to total polytropic efficiency.

It is apparent from Figure 5 that the CFD-predicted total pressure ratio and total-total polytropic efficiency are in accord with the experimental measurement data. The difference between numerical results and experimental data may be due to disc friction and leakage 
flow caused by gaps that are neglected in CFD. Apart from these, the numerical scheme used in CFD also contributes some errors such as the discretization algorithms adopted, the turbulence model employed, and the data transformation method of rotor-stator (impellerdiffuser) interfaces. The error between numerical results and experimental data is less than $3 \%$ under all mass flow conditions. This demonstrates that the numerical method applied is reliable and can be used for further investigation.

\subsubsection{Investigation of Performance with Different Diffusers}

In this section, the aerodynamic performance and internal flow of the centrifugal compressor stage with different diffusers are analyzed according to the unsteady timeaveraged results. Firstly, the influence of different diffusers on the stage performance is investigated. The stage performance curves for different diffusers are revealed in Figure 6. The maximum total pressure-rise of the three types of vaned diffusers occurs around $0.9 Q_{\mathrm{d}}$. With the further decreases in mass flow rate, the total pressure-rise of TVD and HVD decreases faster, while the total pressure-rise of SVD can remain higher than that achieved with TVD and HVD. Moreover, the total pressure-rises of the three vaned diffusers are lower than that of VD, and TVD shows the worst total pressure-rise, which could mean that there is an extensive blocking in the diffuser throat under the near-choke operating point $\left(\varphi=1.2 Q_{\mathrm{d}}\right)$. Next, the effect of the different diffuser configurations on the diffuser performance is studied.

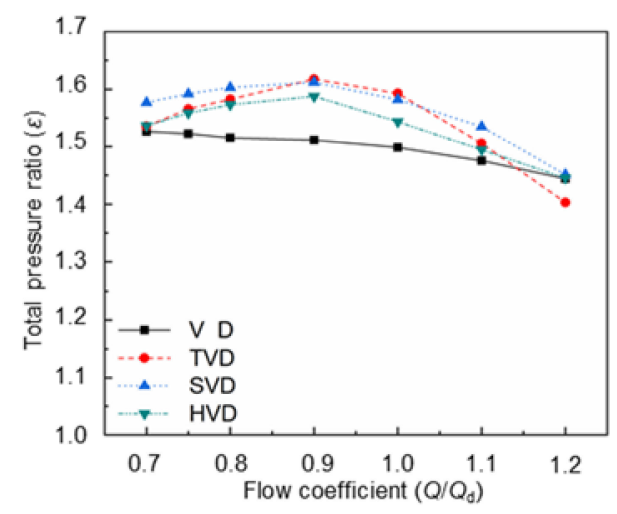

Figure 6. Performance curves for the compressor with different types of diffusers.

The total pressure loss coefficient $\left(C_{\mathrm{pt}}\right)$ and static pressure recovery coefficient $\left(C_{\mathrm{ps}}\right)$ are important indexes for characterizing diffuser performance. The coefficients above are defined as follows [6]:

$$
\begin{gathered}
C_{\mathrm{pt}}=\frac{P_{\mathrm{t} 3}-P_{\mathrm{t} 4}}{P_{\mathrm{t} 3}-P_{\mathrm{s} 3}} \\
C_{\mathrm{ps}}=\frac{P_{\mathrm{s} 4}-P_{\mathrm{s} 3}}{P_{\mathrm{t} 3}-P_{\mathrm{s} 3}}
\end{gathered}
$$

Figure 7 displays the variation of the $C_{\mathrm{pt}}$ and $C_{\mathrm{ps}}$ for different diffusers. At the design point, the minimum $C_{\mathrm{pt}}$ and maximum $C_{\mathrm{ps}}$ are shown by TVD, which means that the loss of TVD is the smallest around design mass flow. However, at the off-design operating point, the total pressure loss of TVD increases rapidly, especially at the low mass flow rate operating point. However, SVD has a lower $C_{\mathrm{pt}}$ and a higher $C_{\mathrm{ps}}$ at low mass flow rate points, and the overall trend is relatively flat between $0.7 Q_{\mathrm{d}}$ and $1.2 Q_{\mathrm{d}}$. This suggests that at the off-design operating point, the performance of TVD deteriorates faster, and SVD has better adaptability. The performance of HVD is almost the same as that of VD, with a slight improvement. It is worth noting that HVD has the most excellent diffuser performance at the near-choke operating point $\left(\varphi=1.2 Q_{d}\right)$. 


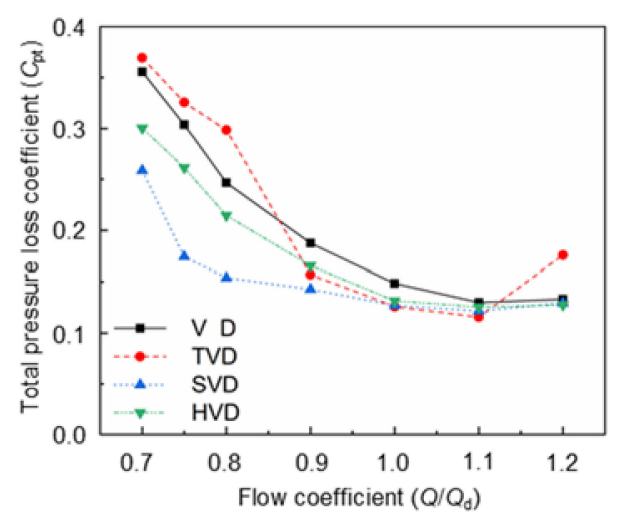

(a)

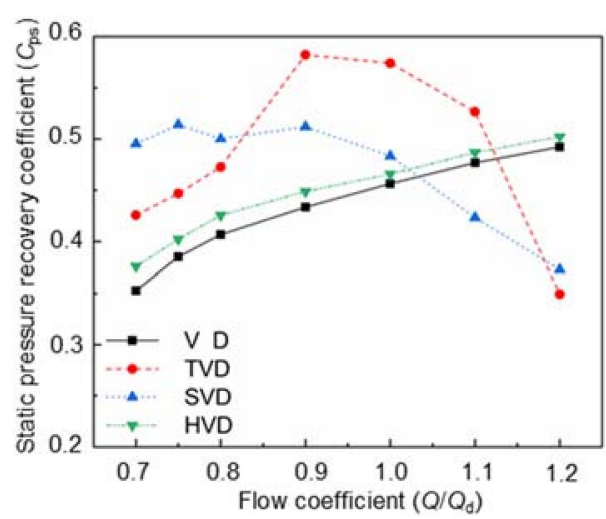

(b)

Figure 7. Performance comparison for different diffusers: (a) total pressure loss coefficient; (b) static pressure recovery coefficient.

\subsubsection{Investigation of the Flow Field in the Different Diffusers}

The flow fields for various diffusers are considered to explore the performance improvement mechanism of different diffuser configurations. It is well known that the operating range of a centrifugal compressor is usually limited by a rotating stall or surge. Therefore, this section focuses on the internal flow conditions in the diffuser at a low mass flow rate point. Figure 8 shows the meridional velocity distribution in different diffusers at $0.7 Q_{\mathrm{d}}$. A sizeable low-momentum flow region is formed near the shroud side in VD. The low-momentum flow region decreases significantly but remains on the shroud side, and the mainstream is accelerated near the hub side in TVD. However, the accelerated mainstream on the hub side is not fully developed downstream of the TVD vane. Similar to $\mathrm{VD}$, the low-momentum flow near the shroud side and the accelerated main steam on the hub side of HVD extend to the diffuser exit. However, the flow in SVD is significantly better than that in the other three diffusers. The low-momentum flow region near the shroud side in VD, TVD, and HVD is well controlled within SVD.

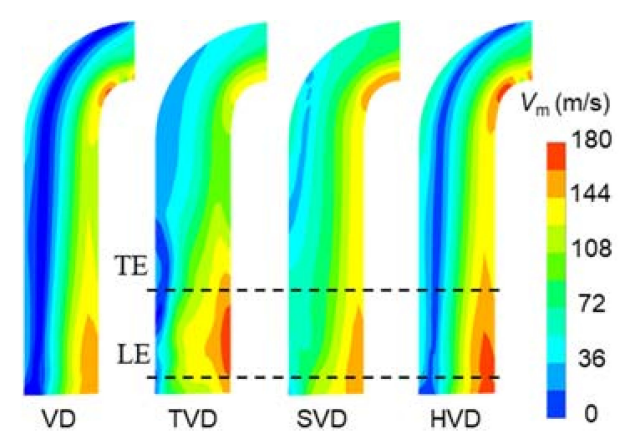

Figure 8. Distribution of meridional velocity at low mass flow rate operating point $\left(\varphi=0.7 Q_{d}\right)$.

Figure 9 shows the static pressure distribution in the meridional direction. Various degrees of low-pressure regions are found at the entrance of different diffusers at $0.7 Q_{\mathrm{d}}$. Similar to the distribution of meridional velocity, HVD and VD have similar static pressure distribution, with a slight improvement. Only a tiny zone of low pressure is found at the entrance of SVD near the shroud side, and the high-pressure region in the meridional direction of SVD is larger than that of the other three types of diffusers. That is the main reason why SVD has the highest static pressure recovery coefficient. 


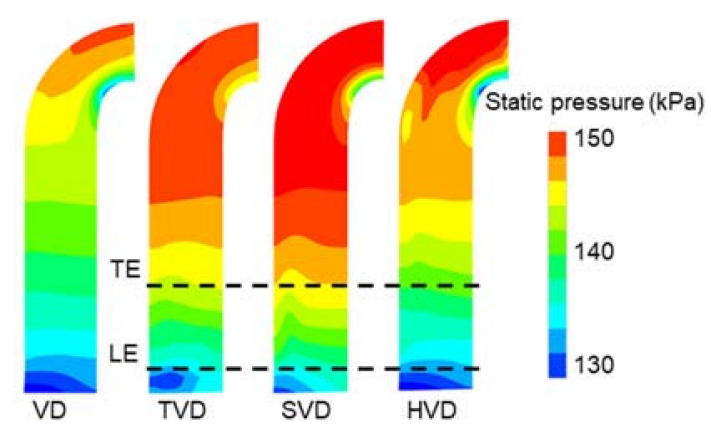

Figure 9. Distribution of static pressure on diffuser meridional direction at low mass flow rate operating point $\left(\varphi=0.7 Q_{\mathrm{d}}\right)$.

To further investigate the performance improvement of SVD compared to TVD, the Mach number distribution at numerous cross-sections of the diffuser passages is shown in Figure 10. As one can see from the TVD, the low-velocity zones formed by large-area flow separation occur in the suction side of the diffuser vane and the diffuser passage. This phenomenon reduces the effective flow area in the diffuser passage, which leads to the acceleration of local flow. However, there is no flow separation phenomenon similar to TVD in the SVD passage. Part of the high-momentum fluid in the SVD passage migrates from the hub side to the shroud side, which suppresses the generation and development of flow separation on the suction side of the diffuser vane. Therefore, SVD can improve the local flow separation phenomenon, and favorable flow characteristics can be attained.

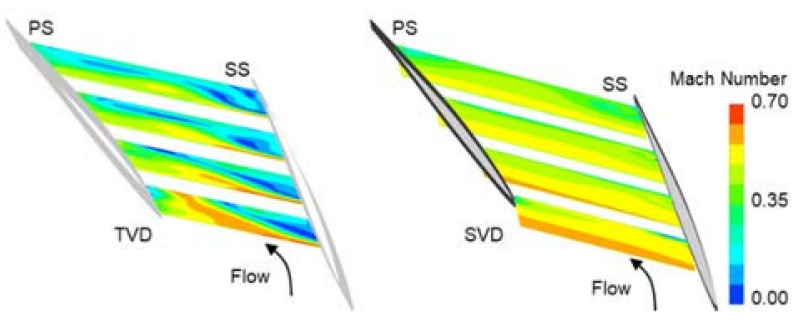

Figure 10. Distribution of Mach number in diffuser passages $\left(\varphi=0.7 Q_{\mathrm{d}}\right)$.

Furthermore, the static pressure distribution in TVD and SVD passages is shown in Figure 11, where the location of the cross-sections in the flow passage is consistent with that in Figure 10. As can be seen from the results of SVD, the low-pressure region mainly appears near the suction side of the diffuser vane. However, the large-scale low-pressure zone appears in the flow passage of TVD. When the fluid flows from the entrance to the diffuser exit, the low-pressure zone in the TVD passage migrates from the middle of the flow passage to the suction surface of the vane. However, the area of the low-pressure region is more prominent than that of SVD. In summary, this is one of the reasons why SVD has favorable pressure recovery characteristics.

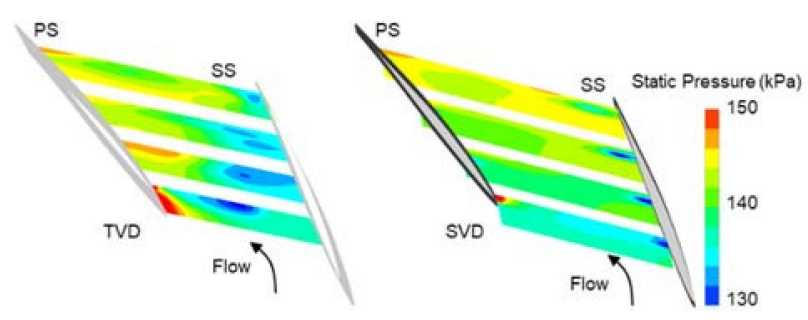

Figure 11. Distribution of static pressure in diffuser passages $\left(\varphi=0.7 Q_{\mathrm{d}}\right)$.

The $Q$-definition [21] is used to visualize the vortex structures, and the nondimensional helicity is used to color the vortex structures. The nondimensional helicity is defined as the 
inner product of vorticity vectors and relative velocity $(-1$ means that the rotation direction of the vortex structure is opposite to that of the impeller, while 1 indicates that the rotation direction of the vortex structure is the same as the rotation direction of the impeller) [9]. A longitudinal vortex is observed on the suction surface of the diffuser, as indicated by a white ellipse dashed line in Figure 12a. The vortex is generated by secondary flow passing through the vane root (indicated by a black arrow in Figure 12b) and is almost stationary. Consequently, the secondary flow caused by this vortex toward the suction surface of the vane (indicated by a white arrow in Figure 12b) prevents the growth of the boundary layer and the development of flow separation on the suction surface of the diffuser vane.

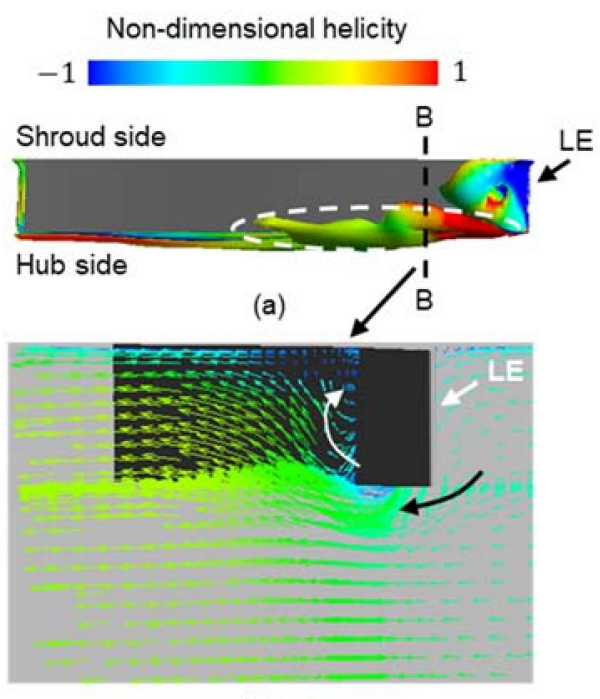

(b)

Figure 12. Vortex and induced secondary flow (SVD, $\varphi=0.7 Q_{\mathrm{d}}$ ): (a) leading-edge vortex of SVD; (b) secondary flow in B-B plane.

The vortex structures of the TVD leading-edge are also obtained using $Q$-definition and colored by nondimensional helicity, as shown in Figure 13. These vortex structures are mainly composed of horseshoe vortices, tornado-like vortices, and transverse vortices. The horseshoe vortices are primarily located at the shroud side and hub side of the diffuser vane inlet. The separation vortex is enlarged due to the relatively low flow angle of the diffuser leading-edge generating a tornado-like vortex that connects the diffuser suctionside surface and shroud wall. Under the action of meridional curvature of the centrifugal impeller blade, low-momentum flow is driven to radial migration from hub side to shroud side [22]. The high adverse pressure gradient reverses flow near the shroud side on the suction surface of the diffuser. Therefore, two transverse vortices (I and II) originate near the shroud side on the suction surface of the diffuser, and the two transverse vortices rotate in opposite directions. Vortex I is clockwise; it is affected by the tornado-like vortex and impacts the diffuser suction surface. Vortex B is counterclockwise; it rotates towards the pressure surface of the adjacent diffuser vane, blocks the mainstream in the diffuser passage, leads to increased flow loss, and even causes the diffuser to stall [23]. Comparing the vortex structures at the leading edges of TVD and SVD, it can be seen that the flow in the passage of the TVD diffuser is more complicated than that in SVD.

\subsection{Unsteady Simulation Results}

According to the aforementioned unsteady time-averaged results, HVD and VD have similar internal flow field and aerodynamic performance, particularly at a low mass flow rate. The unsteady simulation of SVD and TVD was carried out at a low mass flow rate to investigate the internal flow and transient instability evolution processes of the vaned diffuser. 


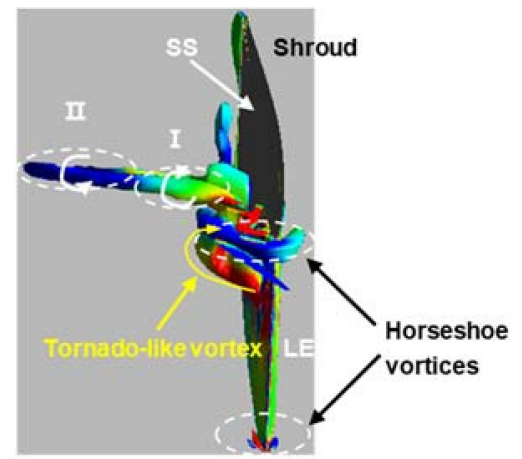

Figure 13. Detailed vortex structures at TVD vane leading-edge $\left(\varphi=0.7 Q_{\mathrm{d}}\right)$.

\subsubsection{Characteristics of Diffuser Stall}

To explore the unsteady behaviors of diffuser stall, the surface static pressure fluctuations were extracted and analyzed on the near shroud side (0.95 span) at seven different locations (Figure 14). The diffuser vane leading-edge (point 1) and middle position of flow passage are distributed equidistantly to the diffuser vane exit (points 2-7). The TVD and SVD spatial distribution of FFT results at different locations under the near-stall operating point are presented in Figure 13. The discrete frequency peaks are observed at $86.645 \mathrm{~Hz}$, the fundamental and two times blade-passing frequency (BPF) in TVD and SVD. The discrete frequency $86.645 \mathrm{~Hz}$, which is approximately equivalent to $35.7 \%$ of impeller-passing frequency (IPF). According to Cumpsty's study [24], the stall frequency is $1 / 5$ to $1 / 2$ of IPF, so $86.645 \mathrm{~Hz}$ was considered the stall frequency of TVD and SVD.

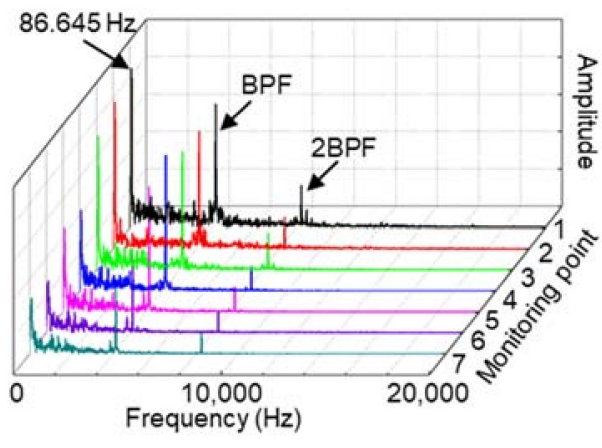

(a)

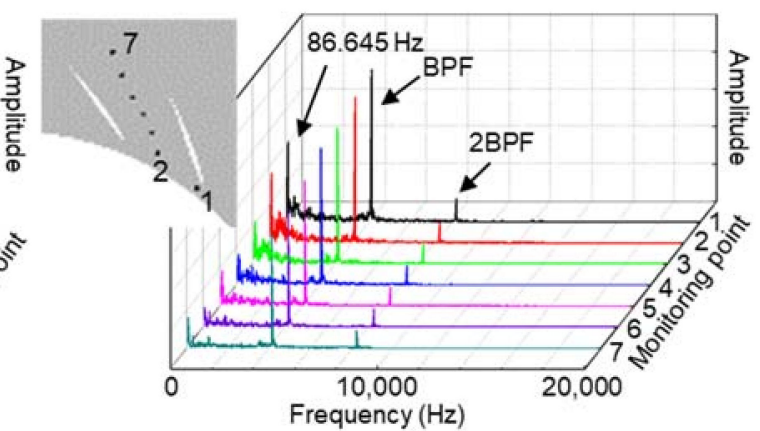

(b)

Figure 14. Spectrum results at different locations $\left(\varphi=0.7 Q_{d}\right)$ : (a) TVD; (b) SVD.

In TVD, the discrete frequency amplitude corresponding to $86.645 \mathrm{~Hz}$ of the diffuser vane inlet (point 1 and point 2) is slightly higher than the fundamental frequency, representing that the pressure fluctuation is more influenced by the diffuser stall cell. As the monitoring point move towards the vane exit, the amplitude of the fundamental frequency is higher than that of the discrete frequency $(86.645 \mathrm{~Hz})$, demonstrating that the pressure fluctuation in diffuser passages is mainly affected by the fundamental frequency. It is noteworthy that at point 3 (diffuser passage throat) of TVD, pressure fluctuation caused by the diffuser stall cells is still significant, which indicates that the diffuser throat of TVD may be blocked by stall cells. The evolution of the leading-edge vortex in the TVD passage will be emphasized below. However, there is no such phenomenon in SVD.

\subsubsection{Unsteady Behavior of Diffuser Stall}

The unsteady behavior of TVD and SVD is presented based on unsteady numerical simulation results to examine the evolution of vortices in the diffuser passages. The unsteady pressure fluctuations at different spans of the TVD entrance (point 1 in Figure 14) are shown in Figure 15. Compared with the unsteady pressure fluctuation at the other 
spans, the pressure fluctuation near the shroud side has a higher amplitude and more complex modal shape.

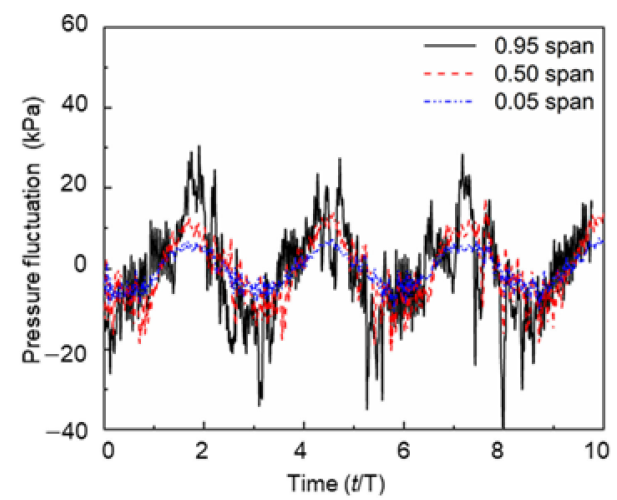

Figure 15. Unsteady pressure fluctuations at the leading edge of TVD $\left(\varphi=0.7 Q_{d}\right)$.

To investigate the evolution of vortices near the diffuser entrance as the stall cell propagates, the instantaneous vortex structures and limiting streamlines were examined, as shown in Figure 16. In these figures, vortex structure behaviors are visualized with $Q$-definition and colored by nondimensional helicity. At time $t=t_{0}$, near the suction surface of the right diffuser vane, only the clockwise-rotating transverse vortex I (Figure 13) is found. Besides, two centers of vortices appear near the suction surface of the vane leadingedge. The counterclockwise-rotating transverse vortex II (in Figure 13) appears at time $t=t_{1}$. Meanwhile, two centers of vortices appearing at time $t=t_{0}$ are merged, making the shroud-side flow more disordered. When the impeller runs to $t=t_{2}$, the transverse vortex II generated from the right diffuser vane increases in size due to the high adverse pressure gradient. The transverse vortex II then moves toward the leading edge of the adjacent left vane and blocks the throat of the diffuser. Note that the flow near the shroud side and leading edge of the right diffuser vane is more disordered, and the flow separation region occupies one-third of the suction surface of the whole vane at time $t=t_{2}$.

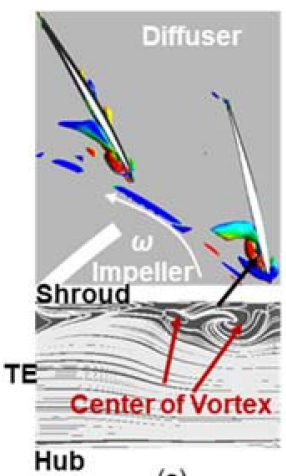

(a)

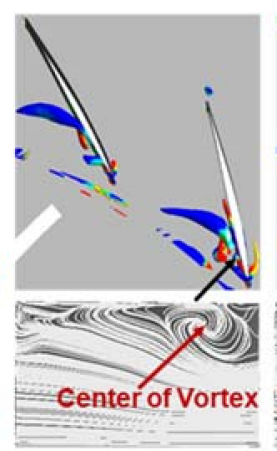

(b)

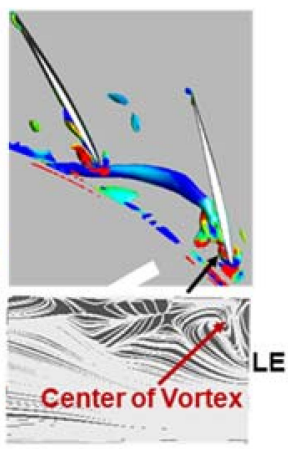

(c)

Figure 16. Unsteady behaviour of vortices (TVD, $\varphi=0.7 Q_{\mathrm{d}}$ ): (a) $t=t_{0} ;(\mathbf{b}) t=t_{1} ;$ (c) $t=t_{2}$.

The pressure fluctuations at 0.95 span of the leading edge of TVD and SVD (point 1 in Figure 14) are compared in Figure 17. Compared with TVD, the amplitude of pressure fluctuation of SVD is decreased sharply. The instantaneous vortex structures of SVD at two moments are shown in Figure 18. Under the action of pressure difference and longitudinal vortex, the high-momentum fluid rotating clockwise through the vane root flows to the shroud side of the suction surface, which prevents the development of the separated flow. It is worth noting that the longitudinal vortex does not propagate into the diffuser passage at the two moments. As can be seen from the limiting streamline figure, in SVD, the vortex scale and its point of origin did not change significantly in two moments. Therefore, the flow field in the SVD passage is relatively stable compared to that of TVD. 


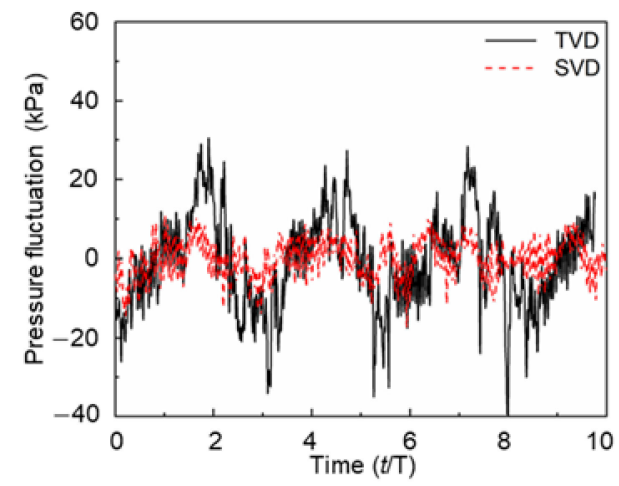

Figure 17. Comparison of unsteady pressure at 0.95 span of diffuser vane leading-edge $\left(\varphi=0.7 Q_{d}\right)$.

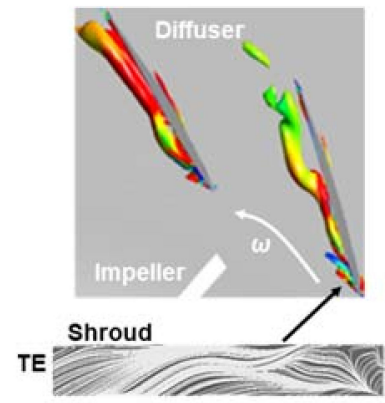

(a)

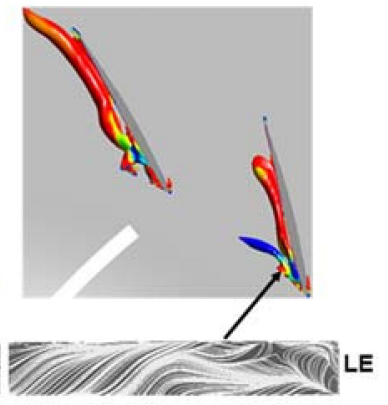

(b)

Figure 18. Unsteady behavior of vortices (SVD, $\left.\varphi=0.7 Q_{\mathrm{d}}\right):(\mathbf{a}) t=t_{\mathrm{s} 0} ;(\mathbf{b}) t=t_{\mathrm{s} 1}$.

\subsubsection{Flow Field Modal Decomposition}

The flow decomposition technique is used to distinguish different flow patterns that occur within a compressor. POD and DMD methods are commonly applied to pressure fields to study the internal flow of fluid machinery in the literature [25-29]. The modal decomposition methods are suitable for the study of pressure loss and flow instability caused by stall cell and impeller-diffuser interaction near the shroud side of the diffuser. With the POD method, the pressure perturbations are not divided into stochastic and deterministic but are represented by modes [30-32]. Then, the large-scale (energetic) flow structures are distinguished by filtering chaos or noise. Next, flow field reconstruction technology reveals the location and magnitude of large-scale flow structures in the flow field. The POD method is utilized to extract a basis of modal decomposition from an ensemble of snapshots to achieve this filtering. A total ensemble of 1000 (about three rotation cycles) instantaneous static pressure flow fields ( 0.95 span in diffuser passage of TVD and SVD) is provided for POD analysis. As the low-order POD modes characterize the energetic flow structures and dominate the flow field, the sensitivity of the ensemble size is tested by comparing low-order POD eigenvalues of each set with data subsets of various sizes. Results indicate that the eigenvalues corresponding to the low-order modes do not change significantly over more than 300 snapshots. Therefore, the ensemble size of snapshots used in this paper far exceeds the ability to provide statistically converging criteria. Finally, the DMD method is used to study the large-scale flow field structure at the special frequencies that have a great influence on the flow field.

Figure 19 shows the eigenvalues corresponding to the first 300 POD modes in TVD and SVD, where the eigenvalues corresponding to each mode are normalized by the firstorder eigenvalue of TVD. Note that since the POD mode exceeds 10 orders, the eigenvalue decreases sharply; the abscissa is in log scale, which provides a statistical representation of smaller-scale flow structures [32]. 


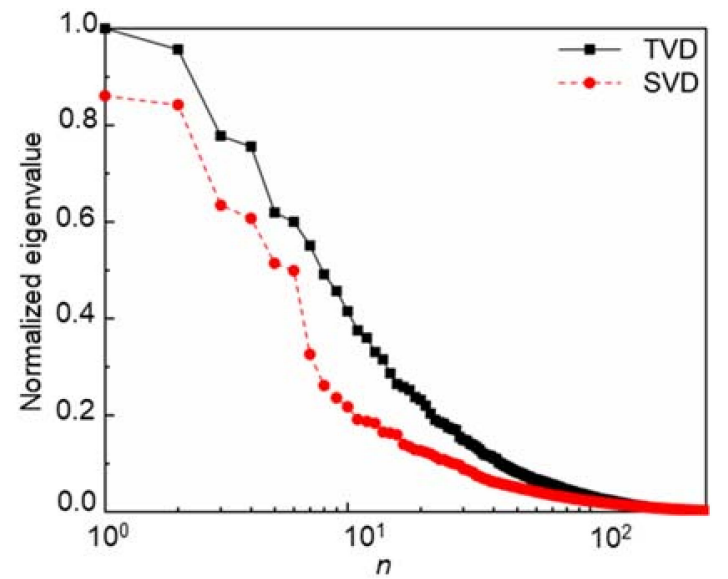

Figure 19. Normalized eigenvalues of the first 300 POD modes.

As can be seen from Figure 19, the energy of POD modes in TVD is higher than that in SVD. Therefore, the POD eigenvalues indicate that large-scale flow structures of TVD possess higher energy compared to that of SVD, which is consistent with the large-scale pressure fluctuation previously observed for diffuser near-stall operating point (in Figure 17).

POD eigenvector (temporal information) analysis further explains the frequency information of large-scale flow structures and distinguishes the different flow patterns more clearly. The eigenvectors corresponding to the first fourth-order POD mode were analyzed by FFT (as shown in Figure 20). For TVD, the diffuser stall frequency $(86.645 \mathrm{~Hz})$ and BPF appear in the first second-order mode, while the third- and fourth-order modes are also correlated with 2BPF. However, for SVD, the third- and fourth-order modes are mainly associated with the fundamental frequency and 2BPF, no longer affected by diffuser stall frequency.

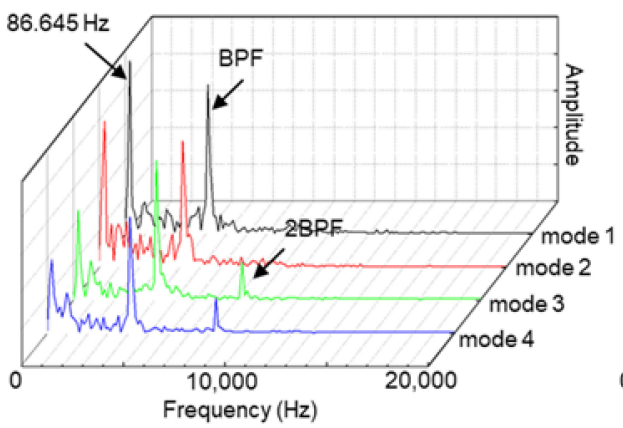

(a)

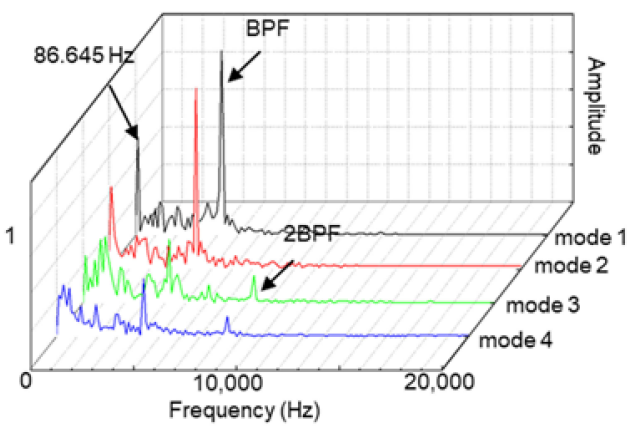

(b)

Figure 20. Spectrum results of POD eigenvectors: (a) TVD; (b) SVD.

The spatial distribution of these POD modes is represented in Figure 21. Each diffuser vane is labeled with numbers from 1 to 4 . Due to periodic boundary conditions, it can be considered that the left-side diffuser passage of vane 1 and the right-side diffuser passage of vane 4 are the same. The zeroth-order POD mode represents the ensemble-averaged flow-field, which includes around $91.176 \%$ of total flow energy, and its spatial pattern is shown in Figure 21a. Compared with TVD, the high static pressure zone near the SVD exit is significantly larger and more uniform. This is the reason why SVD has a higher static pressure recovery coefficient. 


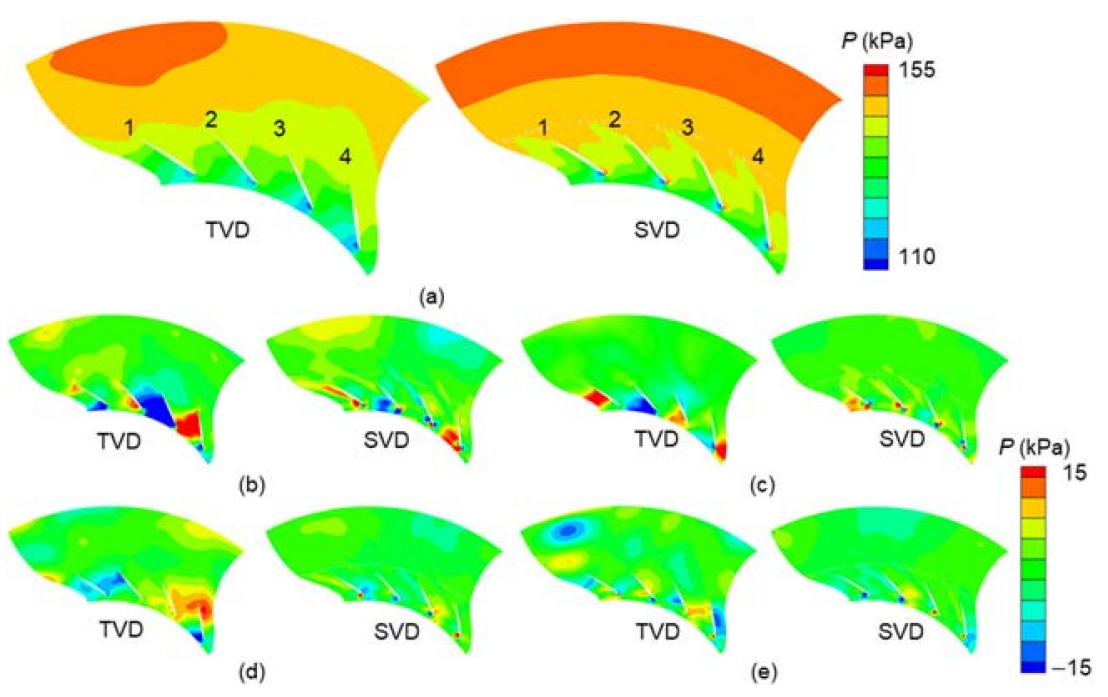

Figure 21. The spatial patterns for POD modes of pressure fields at 0.95 span: (a) zeroth-order POD mode (averaging flow field); (b) 1st-order POD mode; (c) 2nd-order POD mode; (d) 3rd-order POD mode; (e) 4th-order POD mode.

The first second-order POD mode identified large-scale flow structures associated with the diffuser stall cells and BPF. Due to the pseudoperiodic property of POD modes, similar flow structures with similar frequency and energy are observed in the first second-order POD mode [33]. For TVD, the vortex structure formed by stall cells and impeller-diffuser interaction almost blocks two passages of the diffuser, which prevents the fluid from flowing into the passages normally and increases the flow loss in the diffuser. However, a similar diffuser passage blockage phenomenon is not found in SVD, which enables the fluid from the impeller exit to flow into the diffuser normally, thus broadening the stability range of the compressor. The third- and fourth-order POD modes also contributed to understanding the other flow patterns different from the first second-order POD mode. Compared with the first second-order POD mode, the intensity of the vortex structures in the diffuser decreases significantly in the third- and fourth-order POD modes. For TVD, the stall cells still affect the internal flow of the diffuser, which leads to the blockage of some flow passages of the diffuser. However, no similar blockage phenomenon is found in SVD, which further indicates that SVD can improve the stability and performance of the compressor stage.

Based on the POD analysis, the stall frequency $(86.645 \mathrm{~Hz})$ and the blade passing frequency (BPF) have a significant influence on the flow field near the shroud of the diffuser. The pressure field for DMD modes of these two frequencies at 0.95 span is shown in Figure 22. Similar to the POD method, the DMD method is time-dependent, which describes the evolution characteristics of the vortex structures at a specific frequency during a period [29]. It can be seen from Figure 22a that the pressure fluctuation caused by the stall cells mainly appears in the diffuser passages, especially in the TVD, where the largescale vortex structures block part of the diffuser passages. However, the vortex structure caused by the stall cell is weak but still blocks the flow passage of the diffuser, causing the diffuser to stall. The vortex structures caused by the BPF (impeller-diffuser interaction), are mainly distributed at the leading edge of the diffuser vane and present positive and negative alternation (Figure 22b). From an overall point of view, compared with TVD, the intensity of the vortex structures in the diffuser passages in SVD is significantly weakened at two frequencies. This is also the main reason why SVD can improve the stability and performance of the compressor stage compared to TVD. 


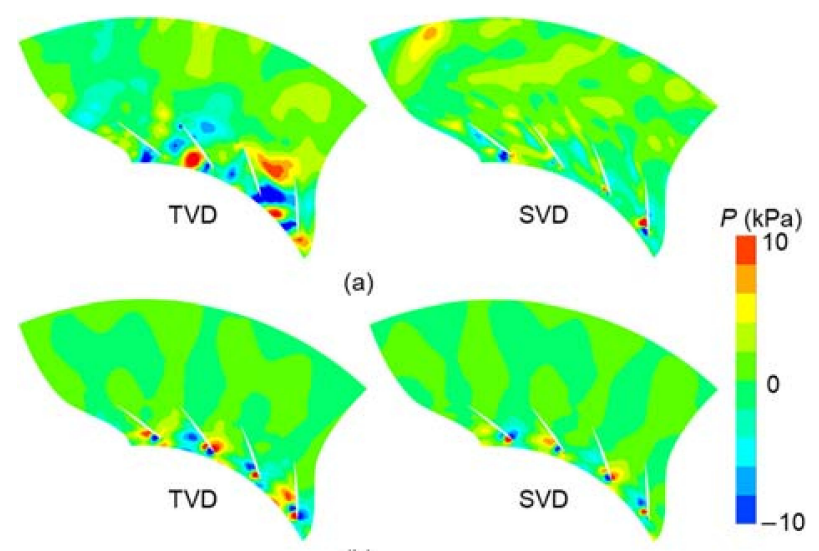

(b)

Figure 22. The pressure fields for DMD modes of two specific frequencies at 0.95 span: (a) $86.645 \mathrm{~Hz}$; (b) BPF.

\section{Conclusions}

Time-averaged unsteady and unsteady numerical simulations have been conducted to analyze the internal flow and aerodynamic performance of a centrifugal compressor with different diffusers. Two vaned diffuser configurations, i.e., TVD and SVD, were mainly investigated at the stall operating point. The purpose of this paper was to clarify the flow field characteristics inside the compressor with different diffusers at the diffuser stall operating point and understand the mechanism of the performance improvement of SVD. Moreover, the unsteady coherent mechanism in TVD and SVD was studied. The main conclusions are summarized as follows:

1. The centrifugal compressor with TVD has the highest total pressure ratio, the minimum $C_{p t}$, and maximum $C_{p s}$ at the design operating point. In contrast, SVD has better flow adaptability, and HVD has the same internal flow characteristics as VD, especially at a low mass flow rate.

2. The mechanism of the improvement in stability by the SVD is that the high-momentum fluid from the vane pressure surface to the suction surface flows to the shroud side through the vane root, which prevents the growth of the boundary layer and the generation of flow separation on the suction surface of the diffuser vane.

3. The rotating stall occurs inside the TVD and SVD diffuser passages at the $70 \%$ design mass flow rate. Results show that the stall cells propagate at $35.7 \%$ of impeller rotational speed in the semi-vaneless space and diffuser passages.

4. The POD and DMD analysis show that the amplitude of pressure fluctuations at the selected cross-section of TVD is higher than that of SVD under the same operating condition. The spatial distribution of the pressure field in the diffuser passages demonstrates that the large-scale flow structure almost blocks the part of diffuser passages, which is also a primary reason for the diffuser stall. Therefore, SVD can improve the stability and performance of the compressor stage when compared to TVD.

Author Contributions: The contribution of all the authors can be subdivided on an equal basis. Conceptualization, validation, writing original draft, visualization, S.L.; validation, resources, writing-review and editing, supervision, Y.L.; project administration, funding acquisition, H.L.; validation, writing - review and editing, M.O. All authors have read and agreed to the published version of the manuscript.

Funding: This research was funded by the National Natural Science Foundation of China, grant number U1808214.

Institutional Review Board Statement: Not applicable.

Informed Consent Statement: Not applicable. 
Data Availability Statement: Not applicable.

Acknowledgments: The authors thanks Shenyang Blower Works Group Corporation for providing the CAD geometry and experimental data for this centrifugal compressor stage.

Conflicts of Interest: The authors declare no conflict of interest.

\section{Nomenclature}

TVD traditional full-height vaned diffuser

VD vaneless diffuser

SVD shroud-side partial vaned diffuser

HVD hub-side partial vaned diffuser

$C_{\mathrm{ps}} \quad$ static pressure recovery coefficient

$C_{\mathrm{pt}} \quad$ total pressure loss coefficient

POD proper orthogonal decomposition

DMD dynamic mode decomposition

$M_{\mathrm{u} 2} \quad$ machine Mach number $\left(M_{\mathrm{u} 2} \sqrt{k R T_{t}}\right)$

$U_{2} \quad$ impeller tip speed $(\mathrm{m} / \mathrm{s})$

$k \quad$ adiabatic exponent

$R \quad$ ideal gas constant $(R=287(\mathrm{~J} / \mathrm{kg} / \mathrm{K}))$

B.C boundary condition

$T_{\mathrm{t}} \quad$ total temperature $(\mathrm{K})$

$Q_{\mathrm{d}} \quad$ mass flow rate at design point $(\mathrm{kg} / \mathrm{s})$

D diameter

LE leading edge

TE trailing edge

BPF blade passing frequency

IPF impeller passing frequency

SS suction surface

PS pressure surface

$t$ time

$\Delta t \quad$ time step

0 centrifugal compressor stage entrance

1 impeller entrance

2 impeller exit

3 diffuser entrance

4 diffuser exit

5 centrifugal compressor stage exit

d design

ps static pressure

pt total pressure

$\lambda$ POD eigenvalue

$\varphi \quad$ mass flow coefficient

$\varepsilon \quad$ total pressure ratio

$\eta_{\text {pol }} \quad$ total-total polytropic efficiency

$\omega \quad$ direction of impeller rotation

\section{References}

1. Clarke, C.; Marechale, R.; Engeda, A.; Cave, M. Investigation of centrifugal compressor vaneless diffuser stability via a local flow angle approach. Proc. Inst. Mech. Eng. Part A J. Power Energy 2016, 230, 366-373. [CrossRef]

2. Anish, S.; Sitaram, N. Computational investigation of impeller-Diffuser interaction in a centrifugal compressor with different types of diffusers. Proc. Inst. Mech. Eng. Part A J. Power Energy 2008, 223, 167-178. [CrossRef]

3. Issac, J.M.; Sitaram, N.; Govardhan, M. Effect of diffuser vane height and position on the performance of a centrifugal compressor. Proc. Inst. Mech. Eng. Part A J. Power Energy 2004, 218, 647-654. [CrossRef]

4. Yoshinaga, Y.; Kaneki, T.; Kobayashi, H.; Hoshino, M. A Study of Performance Improvement for High Specific Speed Centrifugal Compressors by Using Diffusers with Half Guide Vanes. J. Fluids Eng. 1987, 109, 359-366. [CrossRef]

5. Zhang, Y.C.; Kong, X.Z.; Li, F.; Sun, W.; Chen, Q.G. Performance improvement of a centrifugal compressor stage by using different vaned diffusers. IOP Conf. Ser. Mater. Sci. Eng. 2013, 52, 042003. [CrossRef] 
6. Zhao, P.-F.; Liu, Y.; Wang, X.-F. Analysis of vortices and performance of different diffusers in a large mass flow coefficient centrifugal compressor. Proc. Inst. Mech. Eng. Part A J. Power Energy 2017, 231, 253-273. [CrossRef]

7. Liu, R.; Xu, Z. Numerical investigation of a high-speed centrifugal compressor with hub vane diffusers. Proc. Inst. Mech. Eng. Part A J. Power Energy 2004, 218, 155-169. [CrossRef]

8. Sitaram, N.; Issac, J.M. An experimental investigation of a centrifugal compressor with hub vane diffusers. Proc. Inst. Mech. Eng. Part A J. Power Energy 1997, 211, 411-427. [CrossRef]

9. Ohta, Y.; Fujisawa, N. Unsteady behavior and control of vortices in centrifugal compressor. J. Therm. Sci. 2014, 23, 401-411. [CrossRef]

10. Crevel, F.; Gourdain, N.; Moreau, S. Numerical Simulation of Aerodynamic Instabilities in a Multistage High-Speed High-Pressure Compressor on Its Test-Rig_-Part I: Rotating Stall. J. Turbomach. 2014, 136, 101003. [CrossRef]

11. Zamiri, A.; Lee, B.J.; Chung, J.T. Numerical evaluation of transient flow characteristics in a transonic centrifugal compressor with vaned diffuser. Aerosp. Sci. Technol. 2017, 70, 244-256. [CrossRef]

12. Robinson, C.; Casey, M.; Hutchinson, B.; Steed, R. Impeller-Diffuser Interaction in Centrifugal Compressors. In Proceedings of Turbine Technical Conference and Exposition, Parts A and B; ASMEDC: Copenhagen, Denmark, 2012; Volume 8, pp. 767-777.

13. Anish, S.; Sitaram, N.; Kim, H.D. A Numerical Study of the Unsteady Interaction Effects on Diffuser Performance in a Centrifugal Compressor. J. Turbomach. 2014, 136, 011012. [CrossRef]

14. Lumley, J.L. The structure of inhomogeneous turbulence flows. In Atmospheric Turbulence and Radio Wave Propagation; Yaglom, A.M., Tartarsky, V.I., Eds.; Nauka: Moscow, Russia, 1967; pp. 166-177.

15. Sirovich, L. Turbulence and the dynamics of coherent structures. I. Coherent structures. Q. Appl. Math. 1987, 45, 561-571. [CrossRef]

16. Sirovich, L. Turbulence and the dynamics of coherent structures. III. Dynamics and scaling. Q. Appl. Math. 1987, 45, 583-590. [CrossRef]

17. Kim, Y.; Rockwell, D.; Liakopoulos, A. Vortex Buffeting of Aircraft Tail: Interpretation via Proper Orthogonal Decomposition. AIAA J. 2005, 43, 550-559. [CrossRef]

18. Shi, L.L.; Liu, Y.Z.; Wan, J.J. Influence of wall proximity on characteristics of wake behind a square cylinder: PIV measurements and POD analysis. Exp. Therm. Fluid Sci. 2010, 34, 28-36. [CrossRef]

19. Schmid, P. Dynamic mode decomposition of numerical and experimental data. J. Fluid Mech. 2010, 656, 5-28. [CrossRef]

20. Zhao, X.; Zhou, Q.; Yang, S.; Li, H. Rotating Stall Induced Non-Synchronous Blade Vibration Analysis for an Unshrouded Industrial Centrifugal Compressor. Sensors 2019, 19, 4995. [CrossRef] [PubMed]

21. Jeong, J.; Hussain, F. On the identification of a vortex. J. Fluid Mech. 1995, 285, 69-94. [CrossRef]

22. Bousquet, Y.; Carbonneau, X.; Dufour, G.; Binder, N.; Trébinjac, I. Analysis of the Unsteady Flow Field in a Centrifugal Compressor from Peak Efficiency to Near Stall with Full-Annulus Simulations. Int. J. Rotat. Mach. 2014, 2014, 729629. [CrossRef]

23. Fujisawa, N.; Ikezu, S.; Ohta, Y. Structure of Diffuser Stall and Unsteady Vortices in a Centrifugal Compressor with Vaned Diffuser. In Turbo Expo: Power for Land, Sea, and Air; American Society of Mechanical Engineers: New York, NY, USA, 2016. [CrossRef]

24. Cumpsty, N.A.; Greitzer, E.M. A Simple Model for Compressor Stall Cell Propagation. J. Eng. Power 1982, 104, 170-176. [CrossRef]

25. Tinney, C.E.; Jordan, P. The near pressure field of co-axial subsonic jets. J. Fluid Mech. 2008, 611, 175-204. [CrossRef]

26. Liu, H.; Yan, C.; Zhao, Y.; Qin, Y. Analysis of pressure fluctuation in transonic cavity flows using modal decomposition. Aerosp. Sci. Technol. 2018, 77, 819-835. [CrossRef]

27. Sajadmanesh, S.M.; Mojaddam, M.; Mohseni, A.; Nikparto, A. Numerical identification of separation bubble in an ultra-high-lift turbine cascade using URANS simulation and proper orthogonal decomposition. Aerosp. Sci. Technol. 2019, 93, 105329. [CrossRef]

28. Sharma, S.; Broatch, A.; García-Tíscar, J.; Nickson, A.; Allport, J. Acoustic and pressure characteristics of a ported shroud turbocompressor operating at near surge conditions. Appl. Acoust. 2019, 148, 434-447. [CrossRef]

29. Xue, X.; Wang, T.; Shao, Y.; Yang, B.; Gu, C. Experimental and Numerical Analysis of Unsteady Flow Structure in a Centrifugal Compressor with Variable Vaned Diffuser. In Turbo Expo: Power for Land, Sea, and Air; American Society of Mechanical Engineers: New York, NY, USA, 2018. [CrossRef]

30. Semlitsch, B.; Mihaescu, M. Flow phenomena leading to surge in a centrifugal compressor. Energy 2016, 103, 572-587. [CrossRef]

31. Lengani, D.; Simoni, D.; Pichler, R.; Sandberg, R.; Michelassi, V.; Bertini, F. Identification and quantification of losses in a LPT cascade by POD applied to LES data. Int. J. Heat Fluid Flow 2018, 70, 28-40. [CrossRef]

32. Lengani, D.; Simoni, D.; Pichler, R.; Sandberg, R.D.; Michelassi, V.; Bertini, F. On the Identification and Decomposition of the Unsteady Losses in a Turbine Cascade. J. Turbomach. 2019, 141, 031005. [CrossRef]

33. Yarusevych, S.; Kotsonis, M. Effect of Local DBD Plasma Actuation on Transition in a Laminar Separation Bubble. Flow Turbul. Combust. 2017, 98, 195-216. [CrossRef] 